\title{
Correction to: Combined application of electronic nose analysis and back-propagation neural network and random forest models for assessing yogurt flavor acceptability
}

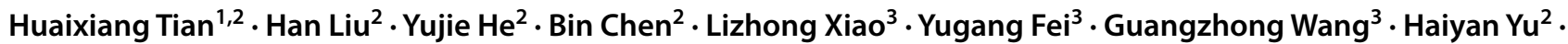 \\ Chen Chen $^{2,4}$ (i)
}

Published online: 29 April 2020

(c) Springer Science+Business Media, LLC, part of Springer Nature 2020

\section{Correction to: \\ Journal of Food Measurement and Characterization (2020) 14:573-583 \\ https://doi.org/10.1007/s11694-019-00335-w}

The original version of the article unfortunately contained an error in one of the affiliations of the first author, Dr. Huaixiang Tian. The institution name was incorrectly published as 'State Key Laboratory of Technology' and the corrected name is 'State Key Laboratory of Dairy Biotechnology'.

This has been corrected with this erratum.

Publisher's Note Springer Nature remains neutral with regard to jurisdictional claims in published maps and institutional affiliations.

The original article can be found online at https://doi.org/10.1007/ s11694-019-00335-w.

Chen Chen

chenchen@sit.edu.cn

1 State Key Laboratory of Dairy Biotechnology, Shanghai Engineering Research Center of Dairy Biotechnology,

Dairy Research Institute, Bright Dairy \& Food Co., Ltd,

Shanghai 200436, China

2 Department of Food Science and Technology,

Shanghai Institute of Technology, 100 Haiquan Road,

Shanghai 201418, China

3 School of Computer Science and Information Engineering, Shanghai Institute of Technology, Shanghai 201418, China

4 Shanghai Research Institute of Fragrance \& Food Flavor, Shanghai 200232, China 